\title{
Nonlinear wood anomalies in the reflectivity of layered superconductors
}

\author{
S.S. Apostolov ${ }^{1}$, Z.A. Maizelis ${ }^{1}$, M.A. Sorokina ${ }^{2}$, and V.A. Yampol'skii ${ }^{1}$ \\ ${ }^{I}$ A.Ya. Usikov Institute for Radiophysics and Electronics, National Academy of Sciences of Ukraine \\ 12 Acad. Proskura Str., Kharkov 61085, Ukraine \\ E-mail: yam@ire.kharkov.ua \\ ${ }^{2}$ V.N. Karazin Kharkov National University, 4 Svoboda Sq., Kharkov 61077, Ukraine
}

Received September 28, 2009

\begin{abstract}
We study theoretically the excitation of the nonlinear surface Josephson plasma waves in slabs of layered superconductors by the incident plane waves using the prism method in the Otto configuration. The bilateral, symmetrical with respect to magnetic field, excitation is considered. The excitation leads to the resonant suppression of the specular reflection, i.e., to the Wood anomalies. Due to the nonlinearity, the reflectivity resonantly depends not only on the wave frequency $\omega$ and the incident angle $\theta$, but on the wave amplitude $h$ as well. We show that the total suppression of the reflectivity can be attained by the appropriate choice of $\omega, \theta, h$, and other parameters of the problem. The results of the analytical calculations are supported by numerical simulations.
\end{abstract}

PACS: 74.78.Fk Multilayers, superlattices, heterostructures;

74.50.+r Tunneling phenomena; Josephson effects;

74.72.-h Cuprate superconductors.

Keywords: layered superconductor, Josephson plasma frequency, gauge-invariant interlayer phase difference of the order parameter.

\section{Introduction}

The recent growing interest in the unusual electrodynamic properties of layered superconductors is due to their possible applications in physics and other fields of modern science, including terahertz ( $\mathrm{THz})$ imaging, astronomy, spectroscopy, health monitoring, environmental control, chemical and biological identification, etc. The artificial stacks of Josephson junctions, e.g., $\mathrm{Nb}-\mathrm{Al}-\mathrm{AlO}_{x}-\mathrm{Nb}$, are the examples of these materials. The strongly anisotropic high- $T_{c} \quad \mathrm{Bi}_{2} \mathrm{Sr}_{2} \mathrm{CaCu}_{2} \mathrm{O}_{8+\delta}$ single crystals are another characteristic members of this family. Intensive experiments for the $c$-axis conductivity in these crystals justify the use of a model in which the very thin superconducting $\mathrm{CuO}_{2}$ layers are coupled by the intrinsic Josephson effect through the thicker dielectric layers [1-5]. Thus, a very specific plasma (so-called, Josephson plasma) is formed in layered superconductors. The current capability of this plasma is strongly anisotropic not only in the absolute values of the current density. Even the physical nature of the currents along and across layers is principally different. The current along the layers is the same as in usual bulk superconductors, whereas the current across the layers has the Josephson origin.
The Josephson current flowing along the $c$-axis couples with the electromagnetic field inside insulating dielectric layers, causing a specific kind of elementary excitations called the Josephson plasma waves (JPW) [6-8]. So, the layered structure of superconductors favors propagation of the electromagnetic waves through the layers. The great challenge is to excite the electromagnetic waves in layered superconductors in a controllable manner because of their $\mathrm{THz}$ frequency range $[9,10]$ which is still hardly reachable for both electronic and optical devices.

The presence of the sample boundary can produce a new branch of the wave spectrum below the Josephson plasma frequency $\omega_{J}$, i.e., for $\omega<\omega_{J}$. These surface Josephson plasma waves (SJPWs) $[11,12]$ are analogs of the surface plasmon polaritons $[13,14]$ in optics. Surface waves play an important role in many fundamental resonance phenomena, such as Wood anomalies in reflectivity $[14,15]$ and transmissivity [16-23] of periodicallycorrugated metal samples.

So far, the Wood anomalies in layered superconductors have been studied for the linear waves only [12]. In this case, the reflection coefficient is controlled by the incident angle and/or the frequency detuning $\left(\omega_{J}-\omega\right)$. At the 
same time, the electrodynamics of layered superconductors is described by the nonlinear coupled sine-Gordon equations [24]. This nonlinearity is originated from a nonlinear relation between the Josephson interlayer current and the gauge-invariant interlayer phase difference of the order parameter. As a result of the nonlinearity, a number of nontrivial nonlinear phenomena, such as the slowing down of light, self-focusing effects, the pumping of weaker waves by stronger ones, etc. $[25,26]$, can be observed in layered superconductors. As was shown in Refs. 8 and 26, the nonlinearity provides the existence of the waveguide modes in slabs of layered superconductors. These nonlinear waveguide modes (NWGMs) are localized inside the slab and exponentially decay away from the superconductor. The dispersion curves, $\omega(q)$, of the NWGMs are below the «vacuum light line», $\omega=c q$, where $q$ is the wave-number and $c$ is the speed of light. This means that the NWGMs have wave-vectors greater than the wavevectors of light of the same frequency in the vacuum. Thus, to excite the NWGMs by means of incident irradiation, it is necessary to use special methods [14], such as the attenuated-total-reflection method.

In this paper, we study theoretically the excitation of the NWGMs in slabs of layered superconductors and the nonlinear Wood anomalies in the slab reflectivity. We consider the Otto experimental configuration where the superconducting slab is separated from the two dielectric prisms by the vacuum gaps (see Fig. 1).

The plain electromagnetic waves propagate in the prisms so that their incident angles $\theta$ exceed the angle $\theta_{t}$ of the total internal reflection. In the absence of the superconductor, the incident waves completely reflect from the bottoms of the prisms. However, the evanescent waves penetrate in the vacuum gaps a distance about a wavelength. The wave-vectors of the evanescent modes are oriented along the bottom surfaces of the prisms and their values are higher than $\omega / c$. This feature is the same as for NWGMs. So, it is natural to expect the spatial-andtemporal matching (coincidence of both, the frequencies and wave-vectors) of evanescent modes and NWGMs for a certain incident angle. This method of the wave excitation is known as the attenuated-total-reflection method $[11,14,27,28]$.

We show in this paper that, due to the nonlinearity, reflectivity of the superconducting slab depends resonantly not only on the wave frequency and the incident angle, but on the wave amplitude as well. Thus, the Wood anomalies can be controlled by the intensity of the incident wave. Moreover, we find the optimal conditions for the total suppression of the specular reflection.

The paper is organized as follows. In Sec. 2, we discuss the geometry of the problem and present the main equations for the electromagnetic field in the dielectric prisms, the vacuum gaps, and the superconductor slab. In Sec. 3, we derive the equation for the reflectivity of the incident

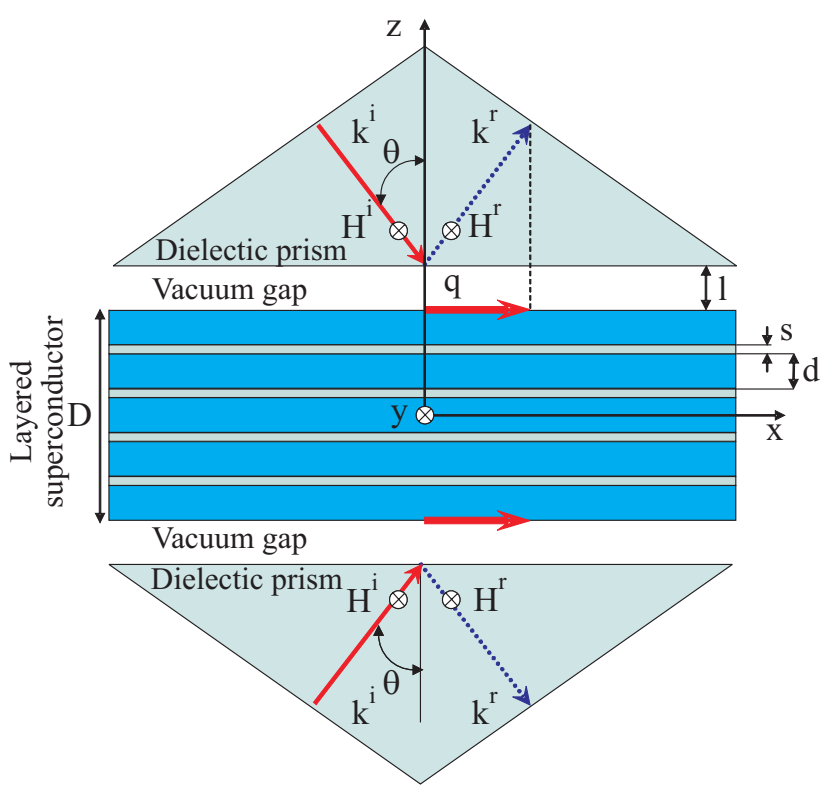

Fig. 1. Geometry for the resonance excitations of NWGMs in a layered superconductor slab of thickness $D$ sandwiched between two dielectric prisms. The latter are separated from a layered superconductor slab by two vacuum gaps of thickness $l$. An electromagnetic wave with incident angle $\theta>\theta_{t}$ can excite SJPWs that satisfy the following resonance condition: $\omega \sqrt{\epsilon_{d}} \sin \theta / c=q$. Here $\mathbf{k}^{i}$ and $\mathbf{k}^{r}$ are the wave-vectors of the incident and reflected waves associated with the magnetic field amplitudes $H^{i}$ and $H^{r}$. The resonance excitation of SJPWs by the incident wave produces a strong suppression of the reflected wave. This method for producing surface waves is known as the «attenuated-total-reflection» method.

wave and analyze its dependence on the incident angle, vacuum gap thickness, and amplitude of the incident wave. The results of numerical simulations support our theoretical predictions.

\section{Distribution of the electromagnetic field}

\subsection{Geometry of the problem}

We study a layered superconducting slab sandwiched between the two dielectric prisms, separated from them by the vacuum gaps of thickness $l$. The thickness $s$ of the superconducting layers is much less than thickness $d$ of insulating layers (see Fig. 1). The coordinate system is chosen in such a way that the crystallographic $a b$-plane coincides with the $x y$-plane and the $c$-axis is along the $z$-axis, the plane $z=0$ corresponds to the middle of the slab. The thickness $D$ of the slab is much greater than the interlayer distance, that allows one to consider the continual limit.

The two plane electromagnetic waves propagate inside the dielectric prisms. We consider the case of transverse magnetic polarization of the waves, when the magnetic field is parallel to the surface of the slab,

$$
\mathbf{E}=\left\{E_{x}, 0, E_{z}\right\}, \quad \mathbf{H}=\{0, H, 0\} .
$$


The incident angle $\theta$ for the two waves exceeds the angle of total internal reflection,

$$
\theta>\theta_{t}=\arcsin \left(\frac{1}{\sqrt{\epsilon_{d}}}\right)
$$

where $\epsilon_{d}$ is the permittivity of the dielectric prisms. The magnetic field in the two waves is assumed to be symmetric with respect to the middle of the slab,

$$
H^{d}(x, y,-z, t)=H^{d}(x, y, z, t) .
$$

This allows us to consider the field distribution for $z>0$ only.

\subsection{Electromagnetic field in dielectric prism and vacuum gap}

The magnetic field $H^{d}$ in the dielectric prism can be represented as a sum of the incident and reflected waves with amplitudes $H^{i}$ and $H^{r}$,

$$
H^{d}=H^{i} \cos \left(q x-k_{d} z-\omega t\right)+H^{r} \cos \left(q x+k_{d} z-\omega t+\alpha\right) .
$$

Here tangential $q$ and normal $k_{d}$ components of the wave-vector for waves in the prism are

$q=k \sqrt{\epsilon_{d}} \sin \theta, \quad k_{d}=\sqrt{k^{2} \epsilon_{d}-q^{2}}=k \sqrt{\epsilon_{d}} \cos \theta$,

$k=\omega / c$, and $\alpha$ is the phase shift of the reflected wave.

When $q>\omega / c$, the Maxwell equations yield an exponential solutions for the magnetic field in the vacuum gap,

$$
\begin{aligned}
H^{v} & =H^{i}\left(h^{+} \cos (q x-\omega t+\beta) \exp \left(k_{v} z\right)+\right. \\
& \left.+h^{-} \cos (q x-\omega t+\gamma) \exp \left(-k_{v} z\right)\right) .
\end{aligned}
$$

Here $h^{ \pm}$are the dimensionless amplitudes of the evanescent waves that exponentially increase/decrease with the growth of $z ; \beta$ and $\gamma$ are their phases, and

$$
k_{v}=\sqrt{q^{2}-k^{2}}=k \sqrt{\epsilon_{d} \sin ^{2} \theta-1}>0 .
$$

Using Maxwell's equations one can express the $x$-components of the electric field in the dielectric prism and vacuum gap via the magnetic field amplitudes,

$$
\begin{gathered}
E_{x}^{d}=-\frac{k_{d}}{k \varepsilon_{d}}\left(H^{i} \cos \left(q x+k_{d} z-\omega t\right)-\right. \\
\left.-H^{r} \cos \left(q x-k_{d} z-\omega t+\alpha\right)\right), \\
E_{x}^{v}=\frac{k_{v}}{k} H^{i}\left(h^{+} \sin (q x-\omega t+\beta) \exp \left(k_{v} z\right)-\right. \\
\left.-h^{-} \sin (q x-\omega t+\gamma) \exp \left(-k_{v} z\right)\right) .
\end{gathered}
$$

Further we find the electromagnetic field in the layered superconductor.

\subsection{Electromagnetic field in the layered superconductor}

The electromagnetic field inside the layered superconductor slab is determined by the distribution of the gaugeinvariant phase difference $\varphi(x, z, t)$ of the order parameter between the layers (see, e.g., Ref. 8),

$$
\begin{gathered}
\frac{\partial H^{s}}{\partial x}=\frac{H_{0}}{\lambda_{c}}\left(\frac{1}{\omega_{J}^{2}} \frac{\partial^{2} \varphi}{\partial t^{2}}+\frac{\omega_{r}}{\omega_{J}^{2}} \frac{\partial \varphi}{\partial t}+\sin \varphi\right), \\
E_{x}^{s}=-\frac{\lambda_{a b}^{2}}{c} \frac{\partial^{2} H^{s}}{\partial z \partial t} .
\end{gathered}
$$

Here $H_{0}=\Phi_{0} / 2 \pi d \lambda_{c}, \Phi_{0}=\pi c \hbar / e$ is the magnetic flux quantum, $\lambda_{a b}$ and $\lambda_{c}=c / \omega_{J} \epsilon_{s}^{1 / 2}$ are the London penetration depths in the in-plane and $c$-directions. Josephson plasma frequency is defined as

$$
\omega_{J}=\sqrt{\frac{8 \pi e d J_{c}}{\hbar \epsilon_{s}}}
$$

where $J_{c}$ is the critical value of Josephson current density, $\epsilon_{S}$ is the permittivity of the dielectric layers in the slab. The relaxation frequency $\omega_{r}=4 \pi \sigma_{c} / \epsilon_{S}$ is proportional to the $c$-axis quasi-particle conductivity $\sigma_{c}$.

The phase difference $\varphi$ obeys the set of coupled sineGordon equations, that, in the continuous limit, take on the form of the following equation (see, e.g., Ref. 29):

$$
\left(1-\lambda_{a b}^{2} \frac{\partial^{2}}{\partial z^{2}}\right)\left[\frac{1}{\omega_{J}^{2}} \frac{\partial^{2} \varphi}{\partial t^{2}}+\frac{\omega_{r}}{\omega_{J}^{2}} \frac{\partial \varphi}{\partial t}+\sin \varphi\right]-\lambda_{c}^{2} \frac{\partial^{2} \varphi}{\partial x^{2}}=0 .
$$

In this paper we consider the case of weak nonlinearity when the Josephson current density $J_{c} \sin \varphi$ can be expanded into series over small $\varphi$ up to the third order, $J_{c} \sin \varphi \sim J_{c}\left(\varphi-\varphi^{3} / 6\right)$. Equation (10) has waveguide solutions for the frequencies $\omega$ lower than $\omega_{J}$. We consider the frequencies $\omega$ close to $\omega_{J}$ and introduce a parameter

$$
\Omega=\frac{\omega}{\omega_{J}}<1
$$

close to one. In this case, in spite of the weakness of nonlinearity in Eq. (10), the linear terms nearly cancel each other, and the term $\varphi^{3}$ plays the crucial role in the problem. Moreover, when the frequency $\omega$ is close to the Josephson plasma frequency, one can neglect the higher harmonics generation.

The dissipation in the slab is considered to be sufficiently small,

$$
\varepsilon=\frac{\omega_{r}}{\omega_{J}\left(1-\Omega^{2}\right)} \ll 1 .
$$

As was shown in Ref. 30, the intralayer quasi-particle conductivity, $\sigma_{a b}$, should also be taken into account if $\omega$ is 
far enough from the Josephson plasma frequency. The contribution of the in-plane conductivity to the dissipation can be easily incorporated in our analysis. However, for the frequency range considered here (close to $\omega_{J}$ ), this contribution is strongly suppressed and can be safely omitted because the relative value of the term with $\sigma_{a b}$ is

$$
\left(\frac{\lambda_{a b}}{\lambda_{c}}\right)^{2} \frac{\sigma_{a b}}{\sigma_{c}}(1-\Omega) \ll 1 .
$$

In fact, the electric field in the superconducting slab has the $z$-component also. This component induces a charge in the superconducting layers when the charge compressibility is finite. This results in an additional interlayer coupling (so-called, capacitive coupling). Such a coupling significantly affects the properties of the longitudinal Josephson plasma waves with the wave-vectors perpendicular to the layers. The dispersion equation for the linear Josephson plasma waves with arbitrary direction of the wave-vectors, taking into account capacitive coupling, was obtained in Ref. 31. According to this dispersion equation, the capacitive coupling can be safely neglected in our case, when the wave-vector has a component $q \gtrsim \omega / c$ along the layers, due to the smallness of the parameter $\alpha=\varepsilon R_{D}^{2} / s D$. Here $R_{D}$ is the Debye length for a charge in a superconductor.

We seek the solution of Eq. (10) in the form,

$$
\varphi=\frac{1}{\left(1-\Omega^{2}\right)^{1 / 2}}\left(A_{0} \sin (q x-\omega t)+A_{1} \cos (q x-\omega t)\right),
$$

with $z$-dependent amplitudes $A_{0,1}$. We introduce the dimensionless $z$-coordinate,

$$
\xi=\frac{\kappa z}{\lambda_{a b}}, \quad \kappa=\frac{\lambda_{c} q}{\left(1-\Omega^{2}\right)^{1 / 2}} .
$$

so that, the normalized half-thickness of the sample is $\delta=D \kappa / 2 \lambda_{a b}$.

Substituting phase difference $\varphi$ in the form of Eq. (11) in Eq. (10), one obtains the following set of equations:

$$
\begin{gathered}
\left(A_{0}-\frac{A_{0}^{3}}{8}\right)^{\prime \prime}=A_{0}, \\
\left(A_{1}-\frac{A_{1} A_{0}^{2}}{8}\right)^{\prime \prime}-\varepsilon A_{0}^{\prime \prime}=A_{1},
\end{gathered}
$$

where prime denotes derivation over $\xi$.

Solving Eqs. (13) and (14), we derive

$$
\begin{gathered}
A_{0}(\xi)=a_{0} \exp \xi+\frac{9}{64} a_{0}^{3} \exp (3 \xi), \\
A_{1}(\xi)=a_{1} \exp \xi+\frac{\varepsilon a_{0}}{2} \xi \exp \xi+ \\
+\frac{9 a_{0}^{2} a_{1}}{64} \exp (3 \xi)+\frac{9 \varepsilon a_{0}^{3}}{128} \xi \exp (3 \xi),
\end{gathered}
$$

with constants $a_{0,1}$.

Thus, we expressed the electromagnetic field in the upper half-space $z>0$ via the amplitudes $H_{i, r}, h^{ \pm}$, and $a_{0,1}$. Below we find the relations between them and analyze the reflective properties of the system under consideration.

\section{Reflectivity of the superconducting slab}

We now proceed to the analysis of the reflectivity $R=H_{r} / H_{i}$ of the incident waves. To this end, we match the tangential components of the electric and magnetic fields at the prism-vacuum and vacuum-superconductor interfaces. This yields the following expression for the reflectivity,

$$
R^{2}=\frac{S+4 p^{2} Y^{2}+4(X-Y)^{2}}{S+4 p^{2} Y^{2}+4(X+Y)^{2}}
$$

where

$$
\begin{gathered}
S=\left(X-X_{0}\right)^{2}\left(p^{-2}-1\right)+2\left(X_{0}^{2}-X^{2}\right), \\
Y=\left(-\frac{A_{1}}{A_{0}}+\frac{8 \varepsilon}{8-A_{0}^{2}}\right) C \cos \theta_{t}, \\
X_{0}=C\left(p \cos \theta_{t}+\zeta\right), \quad X=\zeta-p \cos \theta_{t} .
\end{gathered}
$$

Functions $A_{0,1}$ are taken here at the superconducting slab surface $\xi=\delta$. Parameter

$$
p=\frac{k_{v}}{k \cos \theta_{t}}=\frac{\sqrt{\epsilon_{d} \sin ^{2} \theta-1}}{\cos \theta_{t}}
$$

is determined by the incident angle and is small, $\zeta$ is the surface impedance of the solitary slab in the absence of the dissipations,

$$
\zeta=\Gamma \frac{A_{0}^{\prime}\left(8-3 A_{0}^{2}\right)}{A_{0}\left(8-A_{0}^{2}\right)},
$$

and $\Gamma=\Omega \lambda_{a b} \kappa / \lambda_{c} \epsilon_{s}^{1 / 2}$. Amplitude $A_{0}$ of the field in the superconducting slab can be expressed via normalized amplitude of the incident wave,

$$
H^{i}=H_{0} \frac{1-\Omega^{2}}{\kappa} h,
$$

using the continuity of the tangential components of the electromagnetic field.

Parameter $C$ defines the coupling of the electromagnetic field in the dielectric prism and superconductor slab and is governed by the vacuum gap thickness $l$,

$$
C(l)=\exp \left(-2 k_{v} l\right) \text {. }
$$

The coupling between the prism and superconducting slab leads to the change of the dispersion relation for the NWGMs, that can be found by equating the dominator of Eq. (16) to zero. Equality $X=0$ corresponds to the dispersion relation for NWGM in the layered superconductor 
in the absence of dielectric prism. We consider the case of small values of this parameter, $C \ll 1$. Actually, this region, where the radiation leakage of the excited NWGMs through the prism does not dominate, corresponds to the strongest excitation of the waveguide modes by the incident waves. The coupling results in breaking of the total internal reflection of the electromagnetic waves from the dielectric prism-vacuum gap interface. Due to this coupling, the reflection coefficient $R$ in Eq. (16) becomes less than unity. Moreover, as we show below, the reflection of waves with frequency $\omega<\omega_{J}$ can be totally suppressed by choosing the appropriate value of the incident angle $\theta$ and the amplitude of the magnetic field. This provides a way to control, detect, and filter the $\mathrm{THz}$ radiation.

\subsection{Suppression of specular reflection}

The main contribution to the numerator (and denominator) of Eq. (16) is the term $S(h, \theta, C)$. This leads to the sharp angle dependence of the reflectivity. The reflectivity noticeably differs from one for the angles close to the angle of total internal reflection. Moreover, the deviation must be close to small value $\Gamma^{2} / 2\left(\epsilon_{d}-1\right)^{1 / 2}$. If this condition holds, the reflectivity becomes sensitive to the other parameters of the problem, amplitude $h$ and coupling parameter $C$. This dependence is given by the following expression:

$$
R^{2}=\frac{\left(h-h_{\mathrm{res}}\right)^{2}+B^{2}\left(C-C_{\mathrm{res}}\right)^{2}}{\left(h-h_{\mathrm{res}}\right)^{2}+B^{2}\left(C+C_{\mathrm{res}}\right)^{2}},
$$

where

$$
B=\frac{\varepsilon^{3}\left(C+C_{\mathrm{res}}\right)^{2}}{C C_{\mathrm{res}}^{3}} .
$$

The resonant value of the amplitude and coupling parameter are as follows,

$$
\begin{gathered}
h_{\mathrm{res}}^{2}=128 \frac{\epsilon_{d}^{2}\left(C+C_{\mathrm{res}}\right)^{2}}{C \sqrt{\epsilon_{d}-1}}\left(\theta-\theta_{t}-\frac{\Gamma^{2}}{2 \sqrt{\epsilon_{d}-1}}\right), \\
C_{\mathrm{res}}=\frac{\varepsilon}{8 \epsilon_{d} \Gamma} \sqrt{\epsilon_{d}-1} .
\end{gathered}
$$

Equation (21) describes the pronounced dependence of the reflectivity on the amplitude of the incident wave. When its value is far from $h_{\text {res }}$, the reflectivity is close to one. The minimal value of reflectivity is reached for $h=h_{\text {res }}$,

$$
R_{\min }=\left|\frac{C-C_{\mathrm{res}}}{C+C_{\mathrm{res}}}\right|
$$

Further, varying the thickness of the vacuum gap, one can achieve the total suppression of the reflected wave.
Indeed, the condition $C=C_{\text {res }}$ and Eqs. (20), (23) yield the optimal value of $l$,

$$
l_{\mathrm{opt}}=\frac{1}{2 k \Gamma} \ln \frac{8 \Gamma \delta \epsilon_{d}}{\varepsilon \sqrt{\epsilon_{d}-1}} .
$$

Thus, we have shown that, under certain conditions, the reflectivity of the slab vanishes. These are optimal conditions for the excitation of the NWGMs in the superconducting slab.

\subsection{Results of numerical calculations}

In this subsection, we present the results of numerical calculations that support the conclusions obtained analytically. Equations (13), (14) were solved numerically and then the reflectivity was calculated using Eq. (16).

The dependence of the reflectivity on the incident angle is shown in Fig. 2. The amplitude of the magnetic field of the incident wave is fixed. This plot is obtained for two different values of the vacuum gap thickness. The solid curve corresponds to the situation when the coupling parameter equals to its optimal value, $C\left(l=l_{\text {opt }}\right)=C_{\text {opt }}=$ $=\exp \left(-2 k_{v} l_{\text {opt }}\right)$. Thus, the minimal value of the reflectivity coefficient in this case equals to zero, in agreement with the analytic predictions.

The dependence of the reflectivity on the magnetic field amplitude $h$ of the incident wave is presented in Fig. 3. The data are obtained for the same two values of the vacuum gap thickness, so the minimal value of the reflectivity for the optimal thickness equals to zero. The incident angle for this plot is fixed.

We also illustrated the effect of total suppression of the specular reflection by the distribution of the total magnetic

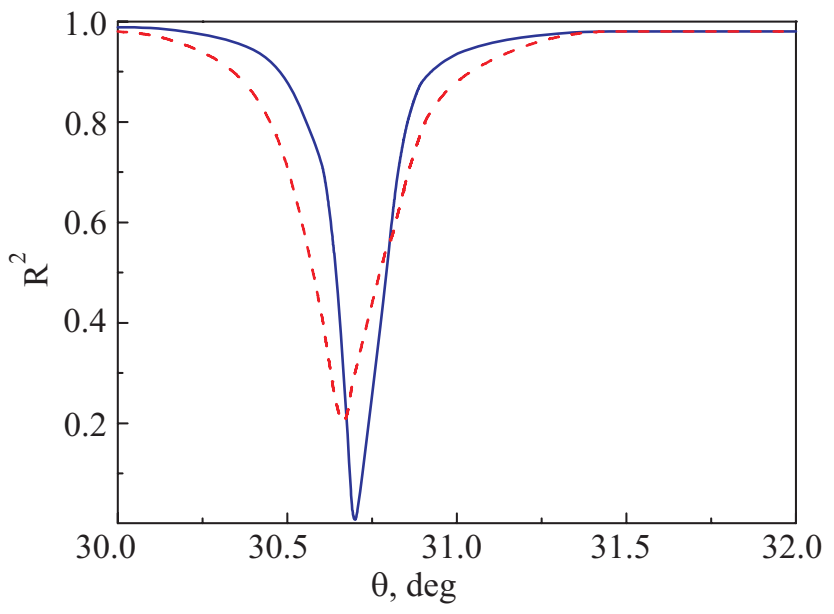

Fig. 2. The dependence of the reflectivity $R^{2}$ on the incident angle $\theta$ for the two different vacuum gap thicknesses: $l=0.04 \mathrm{~cm}$ (dashed curve) and $l=l_{\text {opt }}=0.05 \mathrm{~cm}$ (solid curve). Thus, the minimal value of the reflectivity for the second case equals to zero. The normalized incident wave amplitude is $h=0.14$. Other parameters are: $\lambda_{a b}=2000 \AA, \quad \lambda_{c} / \lambda_{a b}=60, \quad \epsilon_{d}=4$, $\varepsilon=0.05,\left(1-\Omega^{2}\right)=2.5 \cdot 10^{-5}, D / \lambda_{a b}=0.3, \omega_{J}=2 \pi \mathrm{THz}$. 


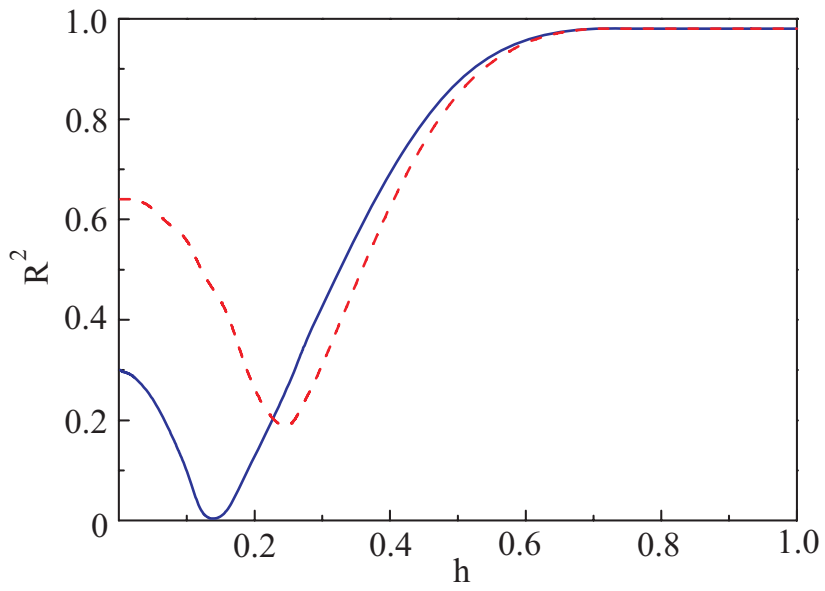

Fig. 3. The dependance of the reflectivity $R^{2}$ on the magnetic field amplitudes $h$ for the incident angle $\theta=30.7^{\circ}$. Other parameters are the same as in Fig. 2.

field in the vacuum and the dielectric prism, Figs. 4 and 5. The interference pattern is seen for the non-resonant case (see Fig. 4), when the amplitudes of the incident and reflected waves practically coincide. Under the resonance condition (see Fig. 4), when the reflected wave is totally suppressed, the interference pattern in the far field disappears, while the near-field «torch» structure of the NWGM is clearly seen near the vacuum-layered superconductor interface.

\section{Conclusion}

Thus, we have studied theoretically the excitation of nonlinear waveguide modes in the layered superconducting slab by the attenuated total reflection method. We have shown that, choosing the optimal combination of the para-

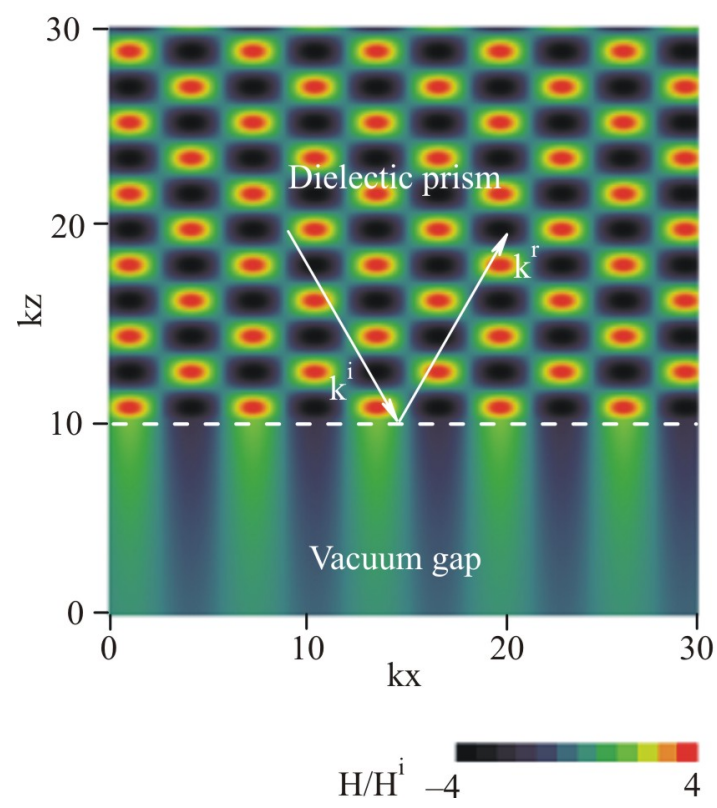

Fig. 4. The magnetic field distribution for the non-resonant case, $h=0.8$, the vacuum gap thicknesses $l=l_{\text {opt }}=0.05 \mathrm{~cm}$. Other parameters are the same as in Fig. 3.

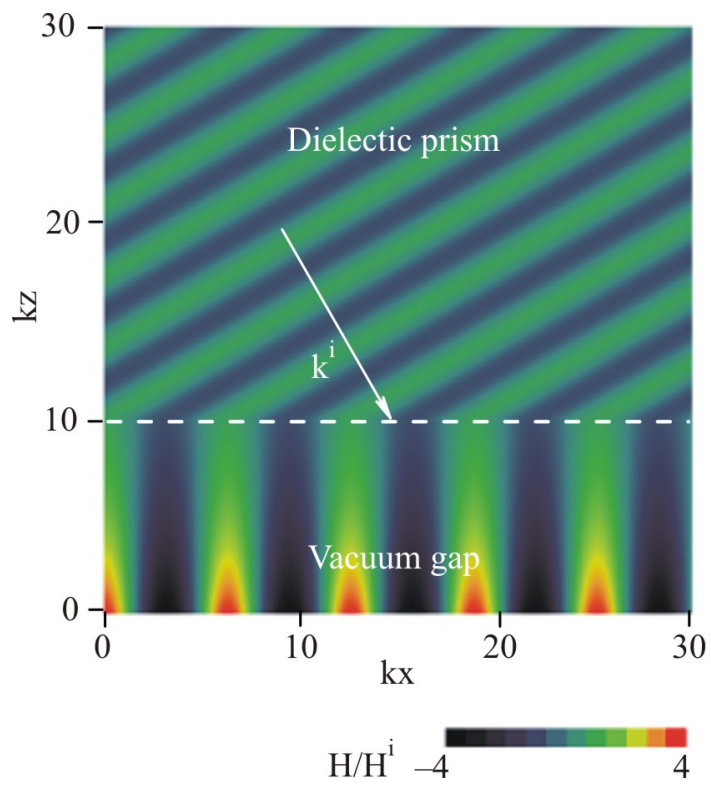

Fig. 5. The magnetic field distribution for the resonant case, $h \simeq 0.13$. Other parameters are the same as in Fig. 4 .

meters of the problem, the reflected wave can be totally suppressed. The equations determining the electromagnetic field in the layered superconductor slab are nonlinear. Due to this nonlinearity, the Wood anomalies can be controlled not only by the incident angle, frequency detuning, and the vacuum gap thickness, but by the amplitude of the incident wave as well. This means that one can fix the wave frequency and the incident angle, and observe vanish of the sample reflectivity, varying the wave amplitude and the vacuum gap thickness. It is important that even small nonlinearity can play the crucial role in the NWGMs excitation due to the cancellation of the displacement and Josephson currents in a superconducting slab. So, even small values of the wave amplitude can be sufficient for suppressing the specular reflection. Indeed, this amplitude is determined by Eq. (19), where $H_{0} \simeq 20$ Oe for $d \simeq 20 \AA$ and $\lambda_{c} \simeq 12 \mathrm{~mm}$. The numerical results are presented for $h \sim 0.1, \quad\left(1-\Omega^{2}\right) / \kappa \simeq 0.5 \cdot 10^{-6}$. At the same time, Eq. (19) shows that the nonlinear Wood anomalies can be observed for very small amplitudes if the frequency detuning $\left(1-\Omega^{2}\right)$ is sufficiently small.

1. R. Kleiner, F. Steinmeyer, G. Kunkel, and P. Müller, Phys. Rev. Lett. 68, 2394 (1992).

2. R. Kleiner and P. Müller, Phys. Rev. B49, 1327 (1994)

3. G. Blatter, M.V. Feigel'man, V.B. Geshkenbein, A.I. Larkin, and V.M. Vinokur, Rev. Mod. Phys. 66, 1125 (1994).

4. E.H. Brandt, Rep. Prog. Phys. 58, 1465 (1995).

5. V.L. Pokrovsky, Phys. Rep. 288, 325 (1997).

6. L.N. Bulaevskii, M.P. Maley, and M. Tachiki, Phys. Rev. Lett. 74, 801 (1995); C.C. Homes, T. Timusk, R. Liang, D.A. Bonn, and W.N. Hardy, Phys. Rev. Lett. 71, 1645 (1993); Y. Matsuda, M.B. Gaifullin, K. Kumagai, K. Kadowaki, T. Mochiku, and K. Hirata, Phys. Rev. B55,

Fizika Nizkikh Temperatur, 2010, v. 36, No. 3 
R8685 (1997); K. Kadowaki, I. Kakea, M.B. Gaifullin, T. Mochiku, S. Takahashi, T. Koyama, and M. Tachiki, Phys. Rev. B56, 5617 (1997); I. Iguchi, K. Lee, and E. Kume, Phys. Rev. 61, 689 (2000); H.B. Wang, P.H. Wu, and T. Yamashita, Phys. Rev. Lett. 87, 17002 (2001); N. Kameda, M. Tokunaga, T. Tamegai, M. Konczykowski, and S. Okayasu, Phys. Rev. B69, 180502(R) (2004).

7. Y. Matsuda, M.B. Gaifullin, K. Kumagai, K. Kadowaki, and T. Mochiku, Phys. Rev. Lett. 75, 4512 (1995).

8. Sergey Savel'ev, V.A. Yampol'skii, A.L. Rakhmanov, and Franco Nori, to be published in: Rep. Prog. Phys.

9. Y. Tominari, T. Kiwa, H. Murakami, M. Tonouchi, H. Wald, P. Seidel, and H. Schneidewind, Appl. Phys. Lett. 80, 3147 (2002).

10. J. Zitzmann, A.V. Ustinov, M. Levitchev, and S. Sakai, Phys. Rev. B66, 064527 (2002).

11. S. Savel'ev, V. Yampol'skii, and F. Nori, Phys. Rev. Lett. 95, 187002 (2005); S. Savel'ev, V. Yampol'skii, and F. Nori, Physica C445-448, 183 (2006); V.A. Yampol'skii, A.V. Kats, M.L. Nesterov, A.Yu. Nikitin, T.M. Slipchenko, Sergey Savel'ev, and Franco Nori, Phys. Rev. B79, 214501 (2009).

12. V.A. Yampol'skii, A.V. Kats, M.L. Nesterov, A.Yu. Nikitin, T.M. Slipchenko, S. Savel'ev, and F. Nori, Phys. Rev. B76, 224504 (2007).

13. P.M. Platzman and P.A. Wolff, Waves and Interactions in Solid State Plasmas, Academic, London (1973).

14. V.M. Agranovich and D.L. Mills, Surface Polaritons, Nauka, Moscow (1985); H. Raether, Surface Plasmons, Springer-Verlag, New York (1988); R. Petit, Electromagnetic Theory of Gratings, Springer, Berlin (1980).

15. A.V. Kats and I.S. Spevak, Phys. Rev. B65, 195406 (2002).

16. A.V. Zayats, I.I. Smolyaninov, and A.A. Maradudin, Phys. Rep. 408, 131 (2005).
17. W.L. Barnes, A. Dereux, and T.W. Ebbesen, Nature (London) 424, 824 (2003).

18. T.W. Ebbesen, H.J. Lezec, H.F. Ghaemi, T. Tio, and P.A. Wolff, Nature (London) 391, 667 (1998).

19. H.F. Ghaemi, T. Thio, D.E. Grupp, T.W. Ebbesen, and H.J. Lezec, Phys. Rev. B58, 6779 (1998).

20. S.C. Hohng, Y.C. Yoon, D.S. Kim, V. Malyarchuk, R.Müller, Ch. Lienau, J.W. Park, K.H. Yoo, J. Kim, H.Y. Ryu, and Q.H. Park, Appl. Phys. Lett. 81, 3239 (2002).

21. L. Martin-Moreno, F.J. Garcia-Vidal, H.J. Lezec, K.M. Pellerin, T. Thio, J.B. Pendry, and T.W. Ebbesen, Phys. Rev. 86, 1114 (2001).

22. A.V. Kats and A.Yu. Nikitin, Phys. Rev. B70, 235412 (2004).

23. A.V. Kats, M.L. Nesterov, and A.Yu. Nikitin, Phys. Rev. B72, 193405 (2005).

24. S. Sakai, P. Bodin, and N.F. Pedersen, J. Appl. Phys. 73, 2411 (1993); L.N. Bulaevskii, M. Zamora, D. Baeriswyl, H. Beck, and J.R. Clem, Phys. Rev. B50, 12831 (1994); S.N. Artemenko and S.V. Remizov, JETP Lett. 66, 811 (1997); S.N. Artemenko and S.V. Remizov, Physica C362, 200 (2001); Ch. Helm, J. Keller, Ch. Peris, and A. Sergeev, Physica C362, 43 (2001); Yu.H. Kim and J. Pokharel, Physica C384, 425 (2003).

25. S. Savel'ev, A.L. Rakhmanov, V.A. Yampol'skii, and F. Nori, Nature Phys. 2, 521 (2006).

26. S. Savel'ev, V.A. Yampol'skii, A.L. Rakhmanov, and F. Nori, Phys. Rev. B75, 184503 (2007).

27. A. Otto, Z. Phys. 216, 398 (1968).

28. H.G. Winful, Phys. Rep. 436, 1 (2006).

29. S. Savel'ev, V. Yampol'skii, A. Rakhmanov, and F. Nori, Phys. Rev. B72, 144515 (2005).

30. A. E. Koshelev, Phys. Rev. B62, R3616 (2000).

31. Ch. Helm and L.N. Bulaevskii, Phys. Rev. B66, 094514 (2002). 